\title{
Novel Technique of Coregistered Intraoperative Computed Tomography and Preoperative Magnetic Resonance Imaging and Diffusion Tensor Imaging Navigation in Spinal Cord Tumor Resection
}

\author{
Tyler Scullen, MD, ${ }^{1,2}$ Jonathan Riffle, DO, ${ }^{1,2}$ Sebastian Koga, MD, ${ }^{1,3,4}$ James Kalyvas, MD ${ }^{1,2,4}$ \\ ${ }^{1}$ Department of Neurological Surgery, Ochsner Clinic Foundation, New Orleans, LA ${ }^{2}$ Tulane University School of Medicine, Tulane \\ University, New Orleans, LA ${ }^{3}$ Division of Neurological Surgery, Ochsner Neuroscience Institute-North Shore Region, Covington, LA ${ }^{4}$ The \\ University of Queensland Faculty of Medicine, Ochsner Clinical School, New Orleans, LA
}

Background: Intradural spinal tumors are surgically challenging lesions, and intraoperative spinal navigation offers clear potential assistance. While intraoperative computed tomography (iCT) of bony anatomy is routinely performed, coregistration with magnetic resonance imaging (MRI) and diffusion tensor imaging (DTI) to facilitate intradural spinal tumor resection is not widely described. We present 2 cases in which iCT was coregistered with MRI and DTI for navigational guidance in the resection of intradural spinal tumors to assess technical feasibility and surgical efficacy.

Case Series: Navigation using coregistered iCT/MRI was used in the resection of one extramedullary and one intramedullary cervicomedullary tumor. The iCT was obtained following open midline exposure of bony anatomy. The images were then coregistered with preoperative MRI sequences to allow for optical tracking navigation via an optical tracking station (Brainlab). For the intramedullary tumor, preoperative DTI sequences were also coregistered for enhanced identification of relevant anatomy. Navigational accuracy for all cases was confirmed to be acceptable at the level of the posterior bony elements, the dura, and the tumor-parenchyma interface.

Conclusion: The coregistration of preoperative MRI sequences and $\mathrm{ICT}$ images allowed for meaningfully enhanced navigation during resection. In the case involving the intramedullary cervicomedullary tumor with marked distortion of longitudinal tracts, iCT/DTI navigation allowed for accurate visualization of critical structures and facilitated delineation of tumor margins that otherwise would have been difficult. The use of combined iCT and preoperative MRI/DTI neuronavigational guidance is an effective approach in the resection of intradural extramedullary and intramedullary spinal cord tumors.

Keywords: Intradural-extramedullary spinal cord neoplasms, neuronavigation, neurosurgical procedures, primary intramedullary spinal cord neoplasms, spinal cord neoplasms

Address correspondence to James Kalyvas, MD, Department of Neurological Surgery, Ochsner Clinic Foundation, 1514 Jefferson Hwy., New Orleans, LA 70121. Tel: (866) 624-7637. Email: james.kalyvas@ochsner.org

\section{INTRODUCTION}

Intradural spinal tumors (ISTs), both intradural extramedullary (IDEM) tumors and intramedullary spinal cord tumors (IMSCTs), represent a rare but challenging disease state secondary to local anatomy and proximity of critical structures within a compact and often restricted operative environment. ${ }^{1-8}$ Preservation of surgical corridors and identification of normal anatomy often necessitate a generous exposure of intradural anatomy, and tumor localization can be difficult-particularly with IMSCTs that are obscured by a normal-appearing spinal cord. ${ }^{8}$ IMSCTs are further complicated by little room for localization error that can lead to profound spinal cord parenchyma damage and devastating complications. ${ }^{1-10}$

The majority of ISTs are benign lesions for which surgical resection remains the frontline therapy. ${ }^{1}$ Spinal cord surgeons must therefore possess a thorough understanding of local anatomy, but often that knowledge is not sufficient, so intradural spinal neuronavigation has a clear potential role in guiding intraoperative decision-making.

Advances in imaging since 2009 have been particularly relevant to the intradural spine. ${ }^{11-19}$ Perhaps the most important is the development of magnetic resonance imaging (MRI) protocols that allow intraoperative coregistration 
of preoperative MRI with intraoperative reconstructed 3dimensional fluoroscopy (3D-fluoro) or intraoperative computed tomography (iCT) on optical tracking navigation systems. ${ }^{2,17,18}$ In the past, spinal navigation was iCT based, and the imaging elucidated primarily bony anatomy only. Coregistration of iCT images with preoperative MRI allows the surgeon to navigate relevant neural anatomy such as the spinal cord and nerve roots, as well as the pathologic anatomy such as a tumor. The advantages of MRI navigation in neurosurgery are well established in cranial surgery. In spine surgery, however, use of this technology is complicated by the mobility of the spine. ${ }^{17}$ Correcting for the differences in patient positioning during preoperative MRI and intraoperative positioning at the time of iCT acquisition is not trivial. Postacquisition curve correction software, whereby each vertebral segment is spatially aligned, is vital for accuracy. ${ }^{17,18}$

The real potential of coregistering preoperative spinal diffusion tensor imaging (DTI) with iCT to visualize tractography during navigation has not been described elsewhere. Although DTI is conceptually valuable in IST surgery, ${ }^{11-15}$ quality spinal DTI is limited by being based on echo-planar imaging and by the spinal cord's high variation in magnetic field homogeneity that can degrade the imaging. ${ }^{17,20}$

We describe cases in which ICT was coregistered with conventional MRI (for an IDEM spinal tumor) and with MRI and DTI (for an IMSCT) to assess the feasibility and surgical efficacy of this navigational technique for IST resection. To our knowledge, this technique has rarely been used in IST resection and never in the treatment of IMSCTs.

\section{CASE SERIES}

Two patients with primary ISTs were treated by a single surgeon (J.K.) at our center in New Orleans, LA, in January 2018. Preoperative MRI was obtained in the form of standard spinal contrast and noncontrast T1- and T2weighted sequences for the first patient and noncontrast T1 magnetization-prepared radiofrequency pulses and rapid gradient-echo sampling (MP RAGE) and DTI sequences for the second patient. MP RAGE sequences constitute the standard stealth protocol used at our facility. Intraoperatively, bony exposures were accomplished via an open midline posterior approach, allowing docking and acquisition of iCT imaging using an Airo mobile iCT scanner (Brainlab). Neuronavigation was then accomplished after coregistering iCT and MRI sequences on an optical tracking station (Brainlab). Navigational accuracy for both cases was confirmed by the surgeon to be acceptable at the level of the posterior bony elements, the dura, and the tumor-parenchyma interface. Final pathologies returned as World Health Organization (WHO) grade 1 primary neuroectodermal tumors in both cases.

\section{Patient 1}

A 28-year-old female with a history of neurofibromatosis type 1 (NF1) and multiple ISTs of the lumbosacral spine presented with progressive low back pain and intermittent left leg pain in an L4 dermatomal distribution. She was clinically followed until an interval MRI showed significant enlargement of an IDEM spinal tumor behind the L3-L4 vertebral bodies with severe canal stenosis (approximately 90\% compromised) and cauda equina compression with intradural

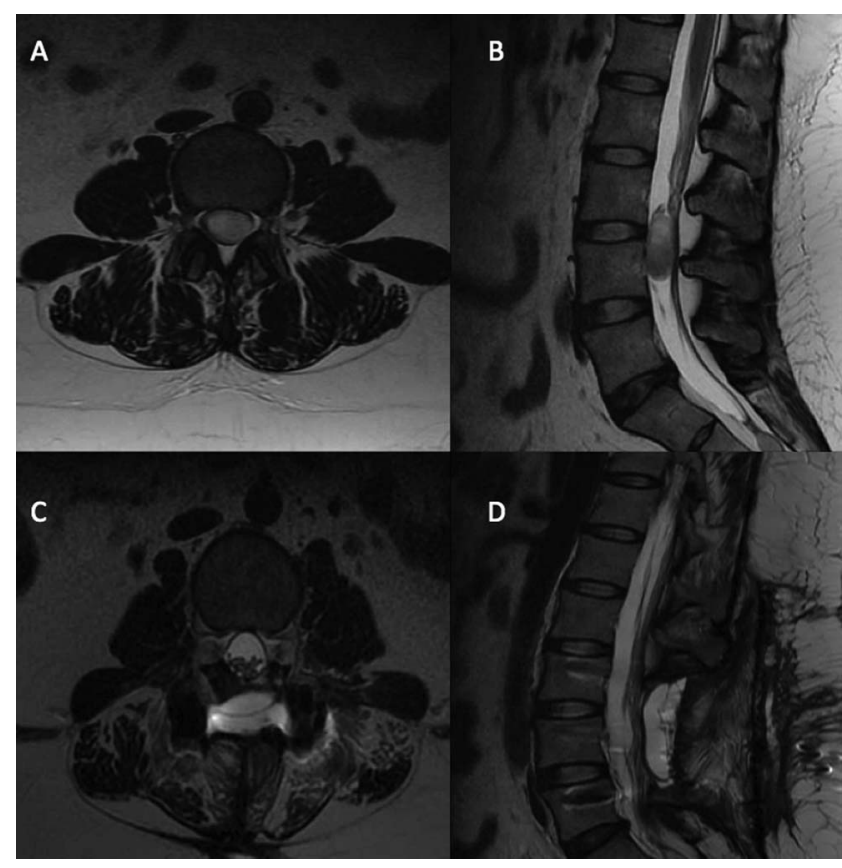

Figure 1. Patient 1: Preoperative (A) axial and (B) sagittal T2-weighted noncontrast fast relaxation fast spin echo magnetic resonance imaging (MRI) of the lumbar spine demonstrates a large intradural extramedullary lesion. Postoperative standard T2-weighted (C) axial and (D) sagittal MRI sequences demonstrate total gross resection.

extension into the left L4-L5 foramen (Figures $1 A$ and 1B). The patient opted for surgical management.

Following exposure of the bony elements from L3-L5, a left L4-L5 medial facetectomy was performed, and the ICT array was docked onto the L5 lamina for image acquisition. Images were coregistered with standard spinal T1- and T2-weighted contrast and noncontrast sequences for navigation and used for intraoperative planning of further bony removal. Laminectomies were performed from L3-L5, along with a left L4-L5 wide foraminotomy to visualize and adequately decompress the exiting left $L 4$ nerve root that was found to be grossly enlarged.

Neuronavigation was again used for planned durotomy and arachnoidotomy for mass exploration and resection. A large IDEM spinal tumor $(1.0 \times 1.5 \times 3.0 \mathrm{~cm})$ was seen in the region of L3-L4, extending out of the left $L 4$ nerve root sleeve with a gross appearance consistent with a peripheral nerve sheath tumor. The mass was removed under gross total resection via subcapsular dissection (Figures 1C and 1D). The involved L4 nerve root was found to have increased stimulation thresholds dorsally. A few adherent dorsal rootlets were divided to decompress the canal adequately, but the ventral roots were preserved. Following intradural resection and closure, the spinal column was stabilized via posterior segmental instrumented fusion (PSIF) and arthrodesis using bilateral freehand pedicle screws and rods from L3-L5. The patient tolerated the procedure well from a hemodynamic and neuromonitoring standpoint. Transcranial motor evoked potentials (MEPs) were present and stable throughout the procedure; left lower extremity somatosensory evoked potentials dropped permanently 


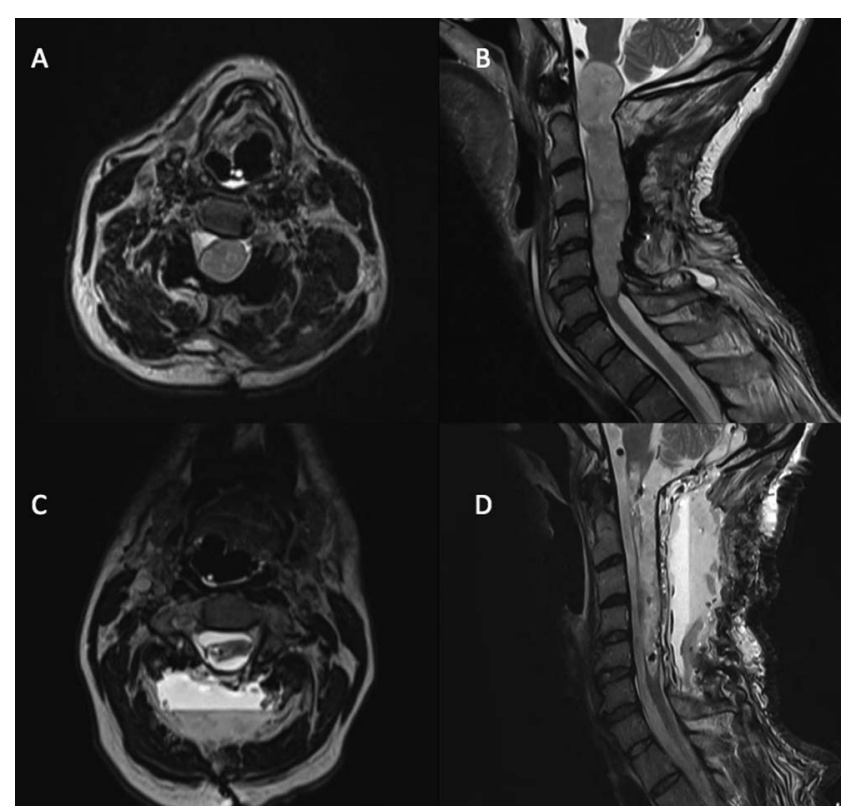

Figure 2. Patient 2: Preoperative T2-weighted noncontrast volumetric 3-dimensional (3-D) magnetic resonance imaging (A) axial and (B) sagittal views of the cervical spine demonstrate a giant intradural intramedullary expansile lesion. Postoperative 3-D T2-weighted (C) axial and standard T2weighted (D) sagittal sequences demonstrate subtotal gross resection of the mass.

approximately $50 \%$ midway through the procedure, which was thought to be secondary to a technical problem in the neuromonitoring circuit rather than attributable to neurophysiologic phenomena. The patient awoke in stable condition with no motor deficits and subjective improvement in radiculopathy, so she was discharged home. Final pathology returned as a spinal neurofibroma, WHO grade 1.

\section{Patient 2}

A 38-year-old male with a history of IMSCT was admitted to our facility with 6 months of progressive spastic quadriparesis. Interval imaging revealed an enlarging expansile mass of the cervicomedullary junction with caudal extension $(2.1 \times 2.4 \times 10.7 \mathrm{~cm})$ across the majority of the cervical cord (Figures $2 \mathrm{~A}$ and $2 \mathrm{~B}$ ). The patient was status post subtotal resection in 2012 with initial residual quadriplegia that slowly resolved to the point of ambulatory status. He remained stable for several years prior to his decline that initially began as profound weakness of the arms and legs, progressed to respiratory dysfunction leading to pneumonias and hypercarbia, and by the time of consultation, his condition had progressed to quadriparesis with global spasticity and contracture of the left arm. Pathology records obtained from the facility of his initial surgery reported findings consistent with spinal subependymoma (WHO grade 1). After extensive discussion, the patient consented to surgical intervention for resection as a life-sparing procedure. Additional preoperative MRI was obtained in the form of noncontrast MP RAGE and DTI sequences for enhanced parenchyma delineation and tractography.

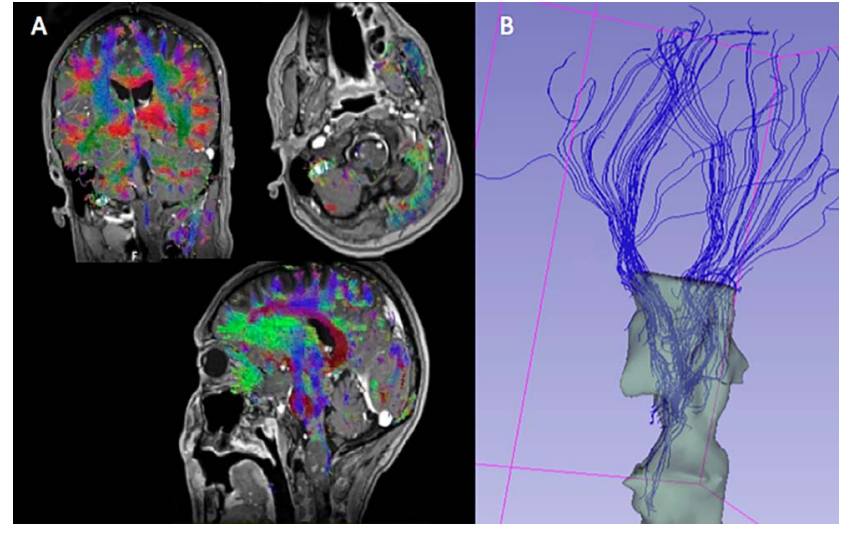

Figure 3. Patient 2: Preoperative (A) diffusion tensor imaging through the brainstem in coronal (top left), axial (top right), and sagittal (bottom) views shows orientation of corticospinal tracts along with (B) 3-dimensional reconstruction showing the positioning of long tracts in relation to a large intramedullary cervicomedullary junction tumor.

Standard open midline bony exposure was carried out from the inion to $\mathrm{C} 6$, incorporating previous PSIF hardware from C2-C5 and laminectomy defects from C1-C5 with areas of pseudarthrosis noted bilaterally. Suboccipital craniectomy and C6 laminectomy were performed to allow adequate decompression and exposure. Baseline MEPs showed signal in the right arm and left leg only.

The CT array was docked onto the Mayfield skull clamp pins (Integra LifeSciences), and iCT imaging was merged with preoperative noncontrast T1-weighted MP RAGE and DTI sequences of the brain, brainstem, and upper cervical spinal cord (Figure 3). Following durotomy at the level of C6, a giant $(2.1 \times 2.4 \times 10.7 \mathrm{~cm})$ IMSCT was seen splaying the cord ventrally and dorsally with exophytic components on either side of the cord and a clear dystrophic appearance of the cord itself. Neuronavigation was used to clarify the tumor-parenchyma interface and localize long tracts. Merged DTI sequences revealed one large tract running dorsally to the lesion with the remaining cord displaced ventrolaterally to the left.

Under neuronavigational guidance, several regions of the mass were entered at the surface bilaterally, and internal debulking was carried out with ultrasonic aspiration. Peripheral dissection revealed good planes around the tumor interface with a resultant subtotal resection of approximately $90 \%$ (Figure 4). During these maneuvers, the patient had an episode of bradycardia and hypertension for several minutes that responded to medication, and MEPs were lost. Dural and soft tissues were closed, and the patient was transferred intubated to the neurocritical care unit in stable condition (Figures 2C and 2D). Immediately postoperatively, the patient remained quadriparetic, regaining only minimal movement in the distal musculature of his left upper extremity. He became ventilator dependent, requiring tracheostomy placement and eventual discharge to a long-term acute care facility. At his 4-month postoperative follow-up, the patient was residing in a skilled nursing facility with minimal but continued interval improvement in neurologic function. Final pathology returned as subependymoma, WHO grade 1. 


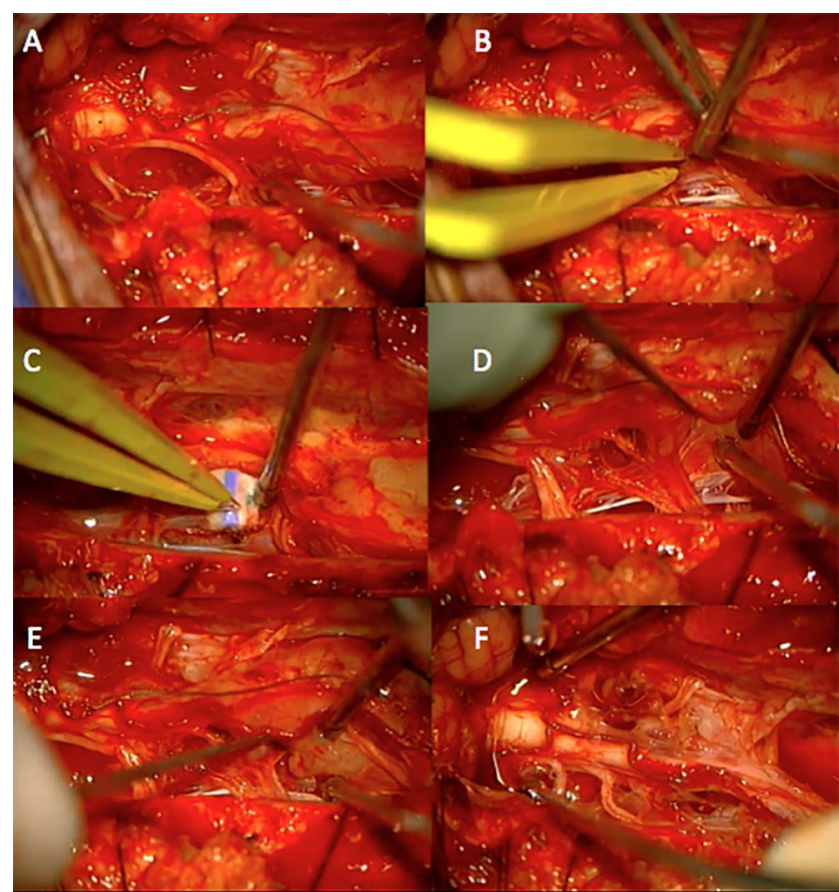

Figure 4. Patient 2: Intraoperative images of microsurgical resection of cervicomedullary subependymoma using merged intraoperative computed tomography and stealth protocol magnetic resonance imaging/diffusion tensor imaging guidance. Following removal of bony elements, the cervicomedullary junction was found to be grossly distorted and dystopic, making identification of normal anatomy difficult (A). Merged navigation was used to identify safe zones for tumor entry (B) and establish dissection planes in the tumor-parenchyma interface (C). The lesion was entered, safely debulked (D), and resected piecemeal (E) with preservation of critical tracts (F).

\section{DISCUSSION}

In this series of 2 cases of surgically managed ISTs, coregistration of preoperative MRI and iCT images allowed for enhanced navigation during resection. In both cases, the surgeon felt that MRI navigation would increase the efficacy of tumor removal while preserving functional outcome.

In the first case, a young patient with known NF1 and multiple intradural lesions correlating with spinal neurofibromas became symptomatic because of an expanding mass at the level of the cauda equina with severe canal compromise and involvement of the $\mathrm{L} 4$ root and dorsal and ventral rootlets. Spinal and plexiform neurofibromas comprise up to $40 \%$ of neoplasms of the NF1 spectrum ${ }^{21}$ and carry a $10 \%-15 \%$ risk of progression to malignant peripheral nerve sheath tumors, ${ }^{22,23}$ particularly those with a deep-seated origin. ${ }^{21}$ As such, rapidly expanding intraspinal lesions with symptom progression become critical targets for preserving quality of life and for delaying the onset of potentially incurable disease. Large IDEM lesions of the cauda equina region present challenges. ${ }^{24}$ Intraoperative spinal navigation with MRI holds promise in aiding tumor localization and thereby minimizing exposure and bony removal, as well as potentially obviating the need for spinal instrumentation. ${ }^{18,25,26}$
In this case, our first experience with the technology, the aim was to establish technical feasibility, and in this regard, the case was successful. We found adequate accuracy at the level of the bone, dura, and the tumor-parenchyma interface. Accuracy was determined by the surgeon based on a comparison with direct visualization of anatomy. Quantifying accuracy will be an important next step. Although curve correction software was not necessary in this case because only 2 spinal segments were involved, we were somewhat limited by the acquisition of too few preoperative MRI slices characteristic of standard spinal protocols. This limitation did not adversely affect this case, but for more complex cases, our recommendation is to acquire thinner preoperative MRI slices to allow more robust coregistration with iCT.

The second case involved a giant expansile recurrent cervicomedullary subependymoma in a young patient with rapidly declining neurologic and functional status. Spinal subependymomas are relatively rare lesions with an uncertain histologic origin and clinical history and with varying theories for treatment options. ${ }^{27-29}$ General recommendations advocate maximum possible excision of symptomatic lesions that will permit preservation of functional status. ${ }^{30,31}$ For cervicomedullary subependymomas with clinical recurrence, the literature provides very little information regarding management. The recommendation for surgery in this particularly challenging clinical scenario was made in the context of a rapidly deteriorating condition in a young male with new-onset compromised respiratory function. As the natural history of his disease was likely fatality, our surgical recommendation was based on the goal of life preservation.

In this case, the role for MRI- and DTI-based navigation was important. The patient had marked distortion of normal neuroanatomy from previous surgery and extensive tumor involvement. Further, localization of longitudinal spinal tracts was quite difficult without DTI. DTI revealed that the bulk of the longitudinal tracts was displaced ventrolaterally to the patient's left side except for one large tract along the dorsum of the tumor. Microscopic visualization and bipolar neurostimulation alone, without navigation, would have been inadequate for safe tumor resection. The addition of merged DTI and stealth sequences with iCT provided a great benefit in intraoperative navigation, allowed for accurate visualization of critical structures, and facilitated delineation of tumor margins. Approximately $90 \%$ of the tumor was removed, an outcome that was not considered likely without DTI navigation - if tumor resection were attempted at all.

This case had some technology limitations. Our institution does not have adequate DTI rendering software for the spinal cord, so brain software extending into the cervicomedullary region was used instead. Nevertheless, the lead surgeon determined adequate accuracy at the parenchymatumor interface compared to direct microscopic visualization. Quantification of accuracy is needed in the future. Distinguishing ventral and dorsal spinal tracts would also be helpful when significant anatomic distortion is present, but to our knowledge, this technology is not yet available.

The use of merged volumetric preoperative MRI with reconstructed $3 \mathrm{D}$-fluoro imaging has been described in the past for the resection of intramedullary spinal metastases. ${ }^{2}$ In addition to the use of merged ICT with traditional spinal MRI for IDEM removal, we describe the use of DTI in 
conjunction with high-resolution gradient echo sequences in the resection of a complicated and extensive IMSCT. To the best of our knowledge, the latter is a novel technique that we found to be a powerful intraoperative adjunct.

\section{CONCLUSIONS}

MRI-based neuronavigation is routinely used in the resection of cranial lesions but is more technically difficult to use for spinal pathology and is therefore rarely used. In this case series, we described the use of iCT merged with both conventional MRI and with stealth protocol MRI and DTI sequences for neuronavigational guidance in the resection of 2 ISTs, one IDEM tumor and one IMSCT. We found this coregistration technique to be a technically feasible and surgically effective approach in both cases, particularly for the IMSCT at the cervicomedullary junction. DTI coregistration allowed for safe tumor resection that otherwise would not have been possible. Significant tumor resections were achieved in both patients, and both patients tolerated the procedures well. We will seek to expand upon this technique in future studies to aid ongoing evolution of IST resection.

\section{ACKNOWLEDGMENTS}

The authors have no financial or proprietary interest in the subject matter of this article.

\section{REFERENCES}

1. Benzel E. The Cervical Spine. 5th ed. Philadelphia, PA: Lippincott Williams \& Wilkins; 2012.

2. Stefini R, Peron S, Mandelli J, Bianchini E, Roccucci P. Intraoperative spinal navigation for the removal of intradural tumors: technical notes. Oper Neurosurg (Hagerstown). $2018 \mathrm{Jul}$ 1;15(1):54-59. doi: 10.1093/ons/opx179.

3. Bhimani AD, Denyer S, Esfahani DR, Zakrzewski J, Aguilar TM, Mehta Al. Surgical complications in intradural extramedullary spinal cord tumors - an ACS-NSQIP analysis of spinal cord level and malignancy. World Neurosurg. 2018 Sep;117:e290-e299. doi: 10.1016/j.wneu.2018.06.014.

4. Dekker SE, Glenn CA, Ostergard TA, et al. Resection of 2 intradural extramedullary cervical spine tumors in a patient with neurofibromatosis type 2: 3-dimensional operative video. Oper Neurosurg (Hagerstown). 2018 Jun 5. doi: 10.1093 /ons/opy149.

5. Noh T, Vogt MS, Pruitt DW, Hummel TR, Mangano FT. Pediatric intramedullary spinal cord tumor outcomes using the WeeFIM scale. Childs Nerv Syst. 2018 Sep;34(9):1753-1758. doi: 10.1007/s00381-018-3831-9. 2018.

6. Ottenhausen M, Ntoulias G, Bodhinayake I, et al. Intradural spinal tumors in adults-update on management and outcome. Neurosurg Rev. 2018 Feb 17. doi: 10.1007/s10143-018-0957-x.

7. Ghasem A, Gjolaj JP, Greif DN, Green BA. Excision of a centrally based ventral intradural extramedullary tumor of the cervical spine through a direct posterior approach. Spinal Cord Ser Cases. 2017 Dec 15;3:17092. doi: 10.1038/s41394-017-0017-8.

8. Ciappetta P, Taurone S, Spoletini M, Artico M. Anteriorly placed tumors to the conus: removal by interradicular window. J Biol Regul Homeost Agents. 2017 Oct-Dec;31(4): 1115-1118.

9. Prestor B, Golob P. Intra-operative spinal cord neuromonitoring in patients operated on for intramedullary tumors and syringomyelia. Neurol Res. 1999 Jan;21(1):125-129.

10. Verla T, Fridley JS, Khan AB, Mayer RR, Omeis I. Neuromonitoring for intramedullary spinal cord tumor surgery.
World Neurosurg. 2016 Nov;95:108-116. doi: 10.1016/j.wneu.2016.07.066.

11. Egger K, Hohenhaus M, Van Velthoven V, Heil S, Urbach H. Spinal diffusion tensor tractography for differentiation of intramedullary tumor-suspected lesions. Eur J Radiol. 2016 Dec;85(12):2275-2280. doi: 10.1016/j.ejrad.2016.10.018.

12. Choudhri AF, Whitehead MT, Klimo P, Montgomery BK, Boop FA. Diffusion tensor imaging to guide surgical planning in intramedullary spinal cord tumors in children. Neuroradiology. 2014 Feb;56(2):169-174. doi: 10.1007/s00234-013-1316-9.214.

13. Liu X, Tian W, Chen $\mathrm{H}$, et al. Advanced neuroimaging in the evaluation of spinal cord tumors and tumor mimics: diffusion tensor and perfusion-weighted imaging. Semin Ultrasound CT MR. 2017 Apr;38(2):163-175. doi: 10.1053/j.sult.2016.07.006.

14. Park EH, Lee YH, Jeong EK, Roh YH, Suh JS. Diffusion tensor imaging focusing on lower cervical spinal cord using 2D reduced FOV interleaved multislice single-shot diffusion-weighted echo-planar imaging: comparison with conventional single-shot diffusion-weighted echo-planar imaging. Magn Reson Imaging. 2015 May;33(4):401-406. doi: 10.1016/j.mri.2015.01.007.

15. Alkherayf F, Arab AF, Tsai E. Conus medullaris teratoma with utilization of fiber tractography: case report. J Neurol Surg Rep. 2015 Jul;76(1):e183-e187. doi: 10.1055/s-0035-1555134.

16. Shtaya $A$, Luong CB, Pereira E. Awake intradural spinal tumor resection; case report and literature review. World Neurosurg. 2018 Jun;114:344-347. doi: 10.1016/j.wneu.2018.03.200.

17. Stroman PW, Wheeler-Kingshott C, Bacon M, et al. The current state-of-the-art of spinal cord imaging: methods. Neuroimage. 2014 Jan 1;84:1070-1081.

doi: 10.1016/j.neuroimage.2013.04.124.

18. Desai B, Hobbs J, Hartung G, et al. Image-guidance technology and the surgical resection of spinal column tumors. J Neurooncol. 2017 Feb;131(3):425-435. doi: 10.1007/s11060-016-2325-4.

19. Acerbi F, Cavallo C, Schebesch KM, et al. Fluorescein-guided resection of intramedullary spinal cord tumors: results from a preliminary, multicentric, retrospective study. World Neurosurg. 2017 Dec;108:603-609. doi: 10.1016/j.wneu.2017.09.061.

20. Heidemann RM, Ozsarlak O, Parizel PM, et al. A brief review of parallel magnetic resonance imaging. Eur Radiol. 2003 Oct;13(10):2323-2337. doi: 10.1007/s00330-003-1992-7.

21. Osborn AG. Osborn's Brain: Imaging, Pathology, and Anatomy. Philadelphia, PA: Lippincott Williams \& Wilkins; 2012.

22. Pollack IF, Mulvihill JJ. Neurofibromatosis 1 and 2. Brain Pathol. 1997 Apr;7(2);823-836.

23. Patil TB, Singh MK, Lalla R. Giant malignant peripheral nerve sheath tumor with cauda equina syndrome and subarachnoid hemorrhage: complications in a case of type 1 neurofibromatosis. J Nat Sci Biol Med. 2015 Jul-Dec 6(2):436-439. doi: 10.4103/0976-9668.160029.

24. McCormick P. Microsurgical resection of cauda equina schwannoma with nerve root preservation. Neurosurg Focus. 2014 Sep;37 Suppl 2:Video 6. doi: 10.3171/2014.V3.FOCUS14267.

25. Maduri R, Bobinski L, Duff JM. Image merge tailored access resection (IMTAR) of spinal intradural tumors. technical report of 13 cases. World Neurosurg. 2017 Feb;98:594-602. doi: 10.1016/j.wneu.2016.05.092.

26. Raco A, Pesce A, Toccaceli G, Domenicucci M, Miscusi M, Delfini R. Factors leading to a poor functional outcome in spinal meningioma surgery: remarks on 173 cases. Neurosurgery. 2017 Apr 1;80(4):602-609. doi: 10.1093/neuros/nyw092. 
27. Bostrom A, von Lehe M, Hartmann W, et al. Surgery for spinal cord ependymomas: outcome and prognostic factors. Neurosurgery. 2011 Feb;68(2):302-308; discussion 309. doi: 10.1227/NEU.0b013e3182004c1e.

28. Lombardi D, Scheithauer BW, Meyer FB, et al. Symptomatic subependymoma: a clinicopathological and flow cytometric study. J Neurosurg. 1991 Oct;75(4):583-538. doi: 10.3171/jns.1991.75.4.0583.

29. Yuh WT, Chung CK, Park SH, Kim KJ, Lee SH, Kim KT. Spinal cord subependymoma surgery: a multi-institutional experience. $J$
Korean Neurosurg Soc. 2018 Mar;61(2):233-242. doi: 10.3340/jkns.2017.0405.001.

30. Bergman TA, Haines SJ. Subependymoma of the cervical spinal cord. A case report of long-term survival. Minn Med. 1991 Nov;74(11):21-24.

31. Zhou S, Xiong J, Pan J, Zhang T, Geng D, Zhang J. Neuroradiological features of cervical and cervicothoracic intraspinal subependymomas: a study of five cases. Clin Radiol. 2016 May;71(5):499.e9-e15. doi: 10.1016/j.crad.2016.01.017.

This article meets the Accreditation Council for Graduate Medical Education and the American Board of Medical Specialties Maintenance of Certification competencies for Patient Care, Medical Knowledge, and Practice-Based Learning and Improvement. 